\title{
J-, H-, and K-band Spectra of Three SU UMa-Type Dwarf Novae
}

\author{
Ryoko Ishioka $^{1}$, Kazuhiro Sekiguchi ${ }^{1}$, and Hiroyuki Maehara ${ }^{2}$ \\ ${ }^{1}$ Subaru Telescope, National Astronomical Observatory of Japan, \\ 650 North A'ohoku Place, Hilo, HI 96720, USA \\ ${ }^{2}$ VSOLJ, Namiki 1-13-4, Kawaguchi, Saitama 332-0034, Japan \\ email:ishioka@subaru.naoj.org
}

\begin{abstract}
We present the results of $J_{-}, H$-, and $K$-band spectroscopy on three SU UMatype dwarf novae with orbital periods of $1.33-1.37 \mathrm{hr}$. We performed the SED fitting for the obtained spectra by assuming a power-law distribution for the accretion disk and using template spectra of late-type dwarfs for secondary star. ASAS 002511+1217.2 and EQ J183926+260409 are WZ Sge-type or WX Cet-type dwarf novae. We found strong water absorption features in their spectra, which are characteristic in late M- or L-type dwarfs. The SED fitting suggests that their secondary contributions to the overall SED are less than one third. We identified the secondary stars as M9 and L1 type dwarfs, which are rather less massive but still normal stars. The spectrum of SDSS J013701-091235 is dominated by the secondary component. Spectral features of this object are similar to those of an early M-type dwarf in spite of its short orbital period. The spectrum of SDSS 0137 strongly suggests that the evolutionary path of this object is different from that of ordinary CVs, and this object is a candidate of EI Psc-like systems.
\end{abstract}

Keywords. stars: dwarf novae,binaries: spectroscopic

\section{Introduction}

Cataclysmic variables (CVs) are close binary systems containing a white dwarf and a late-type star, whose mass is transferred to the white dwarf by Roche-lobe overflow. Secondary stars in CVs are expected to be $G$ - M type dwarfs, and less massive for systems with shorter orbital periods. The evolutionary model of CVs can be tested by comparison with the accurate measurement of secondary stars in CVs.

Near-infrared spectroscopy is effective to obtain information of secondary stars in CVs, because late-type dwarfs have their peak in near-IR, on the other hand, emission from white dwarf and accretion disk become weak there (for a brief review of IR observations, see Howell 2005). Many works succeeded in providing good identifications of secondary stars in long- $P_{\text {orb }} \mathrm{CVs}$, because their secondary stars are luminous enough to detect their absorption features (for examples, Harrison et al. 2005: Harrison et al. 2004a). Secondary stars in short- $P_{\text {orb }}$ CVs might be M or L dwarfs, whose contribution is small even in the IR. Many of the spectroscopic observations yielded only upper limits of secondary stars in short- $P_{\text {orb }}$ systems. Here, we report our infrared spectroscopy of three SU UMa-type dwarf novae to study their secondary stars.

\section{Observations}

Selected objects are ASAS 002511-1217.2, EQ J183926+260409, and SDSS J013701.06-091234.9. ASAS 0025 and J1839 are WZ Sge- or WX Cet-type dwarf nova with a superhump period of $1.37 \mathrm{hr}$ and an orbital period of $1.36 \mathrm{hr}$, respectively. J0137 is 

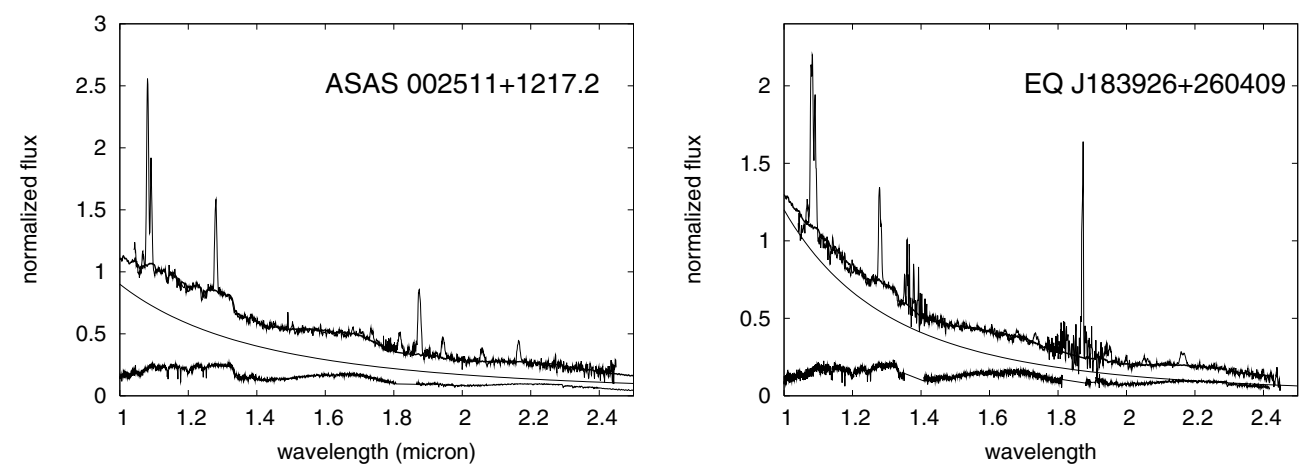

Figure 1. The best SED fit for ASAS 0025 (left) and J1839 (right). The thin and thick lines represent the observed spectra and best-fitted spectra, respectively. Template spectra of M9 and L1 dwarf and power-law components are also presented.

a dwarf nova with an orbital period of $1.33 \mathrm{~h}$. Its optical spectra show Balmer absorption lines of a white dwarf and TiO bandhead of an M-dwarf secondary star.

We performed $J-, H$-, and $K$-band grism spectroscopy using CISCO on SUBARU Telescope. Spectral resolution and exposure time for each band are about 500 and about $1000 \mathrm{sec}$, respectively. We used spectra of A or F type stars obtained with the same configuration for the flux calibration and removal of telluric features.

\section{SED Fitting and Distance Estimation}

Observed spectra are characterized by blue continuum and strong emission lines of $\mathrm{Pa} \alpha$, Bracket series, and HeI $\lambda 1.083 \mu \mathrm{m}$. We performed the spectral energy distribution fitting for the continuum of the spectra to determine the spectral types of secondary stars using the same method as Mennickent \& Diaz (2002). We assumed the continuum of the observed spectrum is composed of a power-law component from the accretion disk and the continuum of a late-type secondary star, and searched the best-fitting template spectrum using the following equation: $S(\lambda)=a T(\lambda)+b \lambda^{c}$, where $S$ and $T$ represent the observed spectra and the template spectra of $\mathrm{M}$ or L-type dwarfs. $\lambda$ is the wavelength and $a, b$, and $c$ are parameters adjusted to minimize the reduced $\chi^{2}$. Template spectra are taken from Cushing et al. (2005).

Figure 1 shows the best-fitted results of the SED fitting for ASAS 0025 and J1839. The continua of these two objects are characterized by strong water absorption features at around $1.35 \mu \mathrm{m}$ and $1.75 \mu \mathrm{m}$. They are well fitted by M7 - L5 dwarfs and power-law components, and best fitted by M9 and L1 dwarfs, respectively. KI and NaI doublets are also detected in the $J$-band spectrum of ASAS 0025. The contribution of the secondary star in each object is $15-20 \%$ in $J$-band and about $50 \%$ in $K$-band.

Figure 2 shows the best SED fit for J0137. The continuum of J0137 does not show strong water absorption features, and is best fitted by an M1 dwarf. There are some hints of absorption lines in the $J$-band spectrum. The contribution of the secondary star is $80-90 \%$, which is consistent with the detection of $\mathrm{TiO}$ bands in optical spectra, but extremely large compared with those of other SU UMa stars.

We estimated distances for our targets in two ways, which are 1) using a distance for the template star and 2) using the empirical relation between orbital periods and magnitudes at outburst maxima: $M_{V}=5.92-0.383 P_{\text {orb }}$ (Warner 1987, Harrison et al. 2004b). Both the $M_{V}-P_{\text {orb }}$ relation and distances for template dwarfs give consistent 

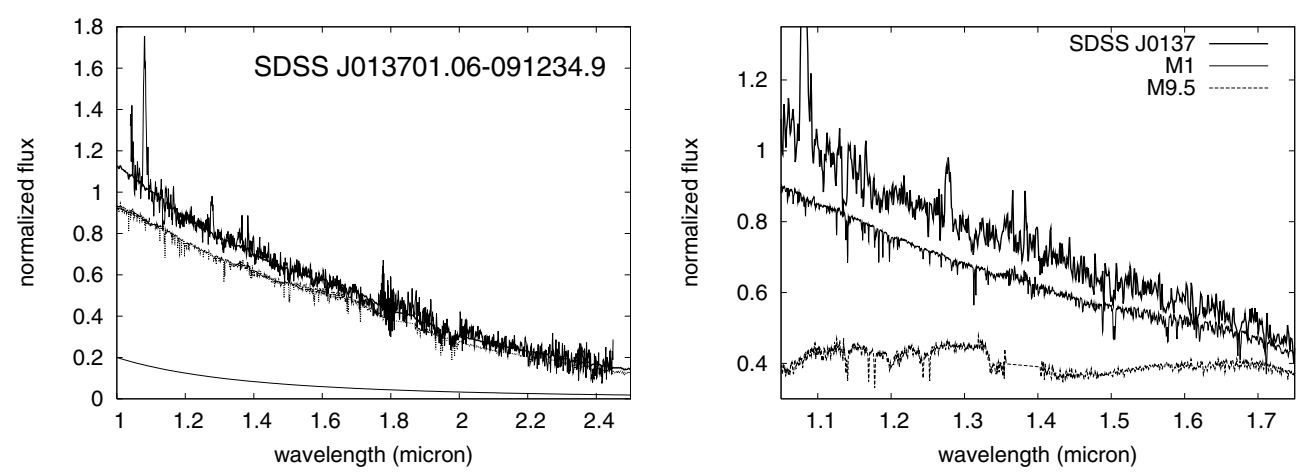

Figure 2. The best SED fit for J0137 (left) and the enhanced spectrum in the $J H$-band (right). The spectrum of J0137 shows some hints of absorption lines characteristic of late-type dwarfs.

distances of $130 \pm 30 \mathrm{pc}$ for ASAS 0025 and J1839. The $M_{V}-P_{\text {orb }}$ relation for J0137 gives a distance of $285 \pm 60$ pc. However, the distance of the M1 dwarf gives a distance of $1200 \pm 300$ pc for J0137.

\section{Conclusion}

The secondary stars of ASAS 0025 and J1839 are classified as M9 and L1 dwarfs. They have less massive secondary stars as suggested from their very short orbital periods and outburst behavior characterized by large amplitudes and long durations. Some CVs with very short orbital periods are expected to have brown dwarfs as secondary stars, but secondary stars of these two objects are still normal stars.

The results on J0137 are rather ambiguous due to the low $S / N$ ratio of our observation. However, the spectrum suggests an early-M dwarf for its secondary star in spite of the short orbital period. The large contribution of the secondary star indicated from our SED fitting is consistent with the $\mathrm{TiO}$ band in optical spectra. The results of distance estimation in two ways are inconsistent with each other. The cause may be that the secondary star in J0137 is rather evolved as in EI Psc.

Our results demonstrate that the SED fitting of the low-resolution infrared spectra is useful to obtain information of the secondary stars of CVs, especially for short-Porb CVs having late-type dwarf secondary stars with strong water absorption features.

\section{Acknowledgements}

We would like to acknowledge the observations by VSNET, AAVSO and VSOLJ members, which we referred to select our targets. This work is supported by the Research Fellowship of the Japan Society for the Promotion of Science for Young Scientists (RI).

\section{References}

Cushing, M.C., Rayner, J.T. \& Vacca, W. D. 2005, ApJ 623, 1115

Harrison, T.E., Johnson, J.J., McArthur, B.E., Benedict, G.F., Szkody, P. Howell, S.B. \& Gelino, D.M. 2004b, $A J$ 127, 460

Harrison, T.E., Obsorne, H.L. \& Howell, S.B. 2004a, AJ 127, 3493

Harrison, T.E., Obsorne, H.L. \& Howell, S.B. 2005, AJ 129, 2400

Howell, S.B. 2005, in A.-M. Hameury \& J.-P. Lasota (ed.), The Astrophysics of Cataclysmic Variables and Related Objects (ASPC) 315, 353

Mennickent, R.E. \& Diaz, M.P. 2002, MNRAS 336, 767

Warner, B. 1987, MNRAS 227, 23 